\title{
Arquitectura Empresarial y Simulación de Procesos. Una Fusión Necesaria antes de realizar Cambios Significativos en la Estructura de Negocio
}

\author{
Jorge E. Puerta-Ramírez, Jaime A. Giraldo-García y Mari L. Tabares-López \\ Universidad Nacional de Colombia, Facultad de Ingeniería y Arquitectura, Departamento de Ingeniería \\ Industrial, Campus La Nubia Bloque Q piso 2, Manizales - Colombia. \\ (e-mail: jepuertar@unal.edu.co, jaiagiraldog@unal.edu.co, mltabaresl@unal.edu.co)
}

Recibido Feb. 20, 2018; Aceptado Abr. 30, 2018; Versión final Jul. 9, 2018, Publicado Feb. 2019

\begin{abstract}
Resumen
El objetivo del estudio que se presenta es comprobar el valor de la fusión entre Arquitectura Empresarial y Simulación de Procesos antes de realizar cambios significativos en la estructura de uno de los macro procesos de la Contraloría General de la República de Colombia. Se identificó el Proceso Auditor como clave para el cumplimiento de la misión de la organización y se utilizó una herramienta de modelado y simulación basada en el estándar Simulación de Procesos de Negocios BPSim (Business Process Simulation) para garantizar una evolución metódica del modelo. El modelado y la simulación mostraron una organización lógica del proceso estudiado y lograron las dimensiones adecuadas para limitar el alcance de la actividad de arquitectura. Con base en los resultados se concluye que la construcción de una Arquitectura Empresarial fusionada con la Simulación de Procesos, genera la posibilidad de crear escenarios para distintas combinaciones de datos, y garantiza una adecuada evolución desde un modelo actual a un modelo objetivo.
\end{abstract}

\section{Business Architecture and Process Simulation - A necessary merger before making significant changes in the business structure}

\begin{abstract}
The objective of the study presented in this paper is to verify the value of the merging between Enterprise Architecture and Process Simulation before making significant changes in the structure of one of the macro processes of the General Comptroller of the Republic of Colombia. The Audit Process was identified as a key for the fulfillment of the mission of the organization. A modeling and simulation tool based on the BPSim standard (Business Process Simulation) was used to guarantee an evolution of the model. The modeling and simulation achieved a logical organization of the process studied and reached the appropriate dimensions for the scope of the activity of the architecture. Based on the results, it is concluded that the construction of a Business Architecture merged with Process Simulation, generates the possibility of creating scenarios for different combinations of data, and guarantee adequate transformation from a current model to an objective model.
\end{abstract}




\section{INTRODUCCIÓN}

La Ley 42 de 1993, instituye a la Contraloría General de la República (CGR) como la entidad fiscalizadora superior del Estado colombiano, constituyéndose en un órgano de control de carácter técnico, con autonomía administrativa y presupuestal para administrar sus asuntos en los términos y en las condiciones establecidas en la Constitución y en las leyes. (CGR, 2016). El Decreto 267 de 2000, determina como objetivos de la CGR, ejercer en representación de la comunidad, la vigilancia de la gestión fiscal de la administración y de los particulares o entidades que manejen fondos o bienes de la Nación; evaluar los resultados obtenidos por las diferentes organizaciones y entidades del Estado en la correcta, eficiente, económica, eficaz y equitativa administración del patrimonio público, de los recursos naturales y del medio ambiente; generar una cultura del control del patrimonio del Estado y de la gestión pública; así mismo la Ley 610 de 2001, establece las responsabilidades fiscales y la imposición de las sanciones pecuniarias que correspondan y las demás acciones derivadas del ejercicio de la vigilancia fiscal. (CGR, 2017). Bajo estas premisas la CGR tiene una serie de retos políticos, jurídicos y de gestión, siendo éste último un factor importante a la hora de demostrar su eficiencia y efectividad en el Control Fiscal, que como lo afirman Petro y Gardiner (2015), Peterson (2016) y Restrepo (2017), debe ser identificado a través del desarrollo de una arquitectura propia para la organización, la cual se puede alcanzar según Pylypenko y Lytvynenko (2017) mediante la aplicación de metodologías de diseño institucional y arquitectónico del desarrollo organizacional.

Del mismo modo, actualmente las organizaciones tienen la necesidad de evaluar su funcionamiento y comportamiento en términos de Arquitectura Empresarial, lo que se convierte en un insumo importante a la hora de tomar decisiones estratégicas y de realizar cambios significativos en la estructura de negocio. Al respecto Cáceres y Zea (2014), Lange y Recker (2016) y Kotusev (2017) mencionan que la Arquitectura Empresarial ha evolucionado de ser una disciplina netamente técnica que visualiza el desempeño de la infraestructura tecnológica de una empresa a convertirse en una disciplina gerencial que permite visualizar el estado actual de una organización a través de modelos (actuales) y evaluar la dirección estratégica, planteando arquitecturas y modelos de escenarios (futuros) para una mejor toma de decisiones en cuanto a: i) estandarización de procesos; ii) flexibilización de la organización; iii) facilidad para la transformación y restructuración del negocio; para con ello mejorar la satisfacción de las partes interesadas. De la misma manera Acosta y Espino (2014) y Grant (2016) indican que el uso de técnicas para describir y analizar los procesos de negocios de una organización, permiten comprender el negocio antes de considerar alguna decisión.

Adicional al uso de técnicas de modelado, para la construcción de una Arquitectura Empresarial, se hace necesario aplicar un marco de referencia (framework), que le permita actuar como base para estructurar y ensamblar los componentes de la organización en estructuras más complejas, determinando los términos a definir y como documentar la aplicación de metodologías del diseño arquitectónico para el desarrollo organizacional. Como consecuencia de lo anterior y teniendo en cuenta lo comentado por Crespo (2015), en cuanto a que la Arquitectura Empresarial, no solo describe como una organización opera funcionalmente, sino que también define y describe los procesos y objetivos de negocio necesarios para la implantación de la estrategia organizacional; y después de considerar las diferentes técnicas de Arquitectura de Empresarial identificadas por Cuenca (2008), Plattner (2014) y Bork (2014), tales como CIMOSA, GRAI-GIM, PERA, GERAM, ARIS, ZACHMAN y TOGAF, para el desarrollo de la investigación, se seleccionó el marco de referencia TOGAF (The Open Group Architecture Framework), el cual incluye el Método de Desarrollo de la Arquitectura (ADM) que comprende: guías, técnicas y modelos de referencia, siendo éste el más completo y riguroso en la definición de los flujos de trabajo.

Ante esto autores como Geyer y Fourie (2015), argumentan que las organizaciones deben definir y documentar sus procesos y los flujos de trabajo con el fin entenderlos y así permitir un mejoramiento continuo de su gestión. Por lo tanto y atendiendo la Guía de Auditoría AUDITE de la CGR, la cual establece las actividades y procedimientos para realizar el proceso auditor, se estima que un punto neurálgico y del cual dependen el rumbo y los resultados del proceso, es la fase de planeación, la cual tiene como objetivo definir el alcance y la estrategia de auditoría a partir del conocimiento del auditado y de la evaluación de los controles que el ente objeto de control fiscal tiene establecidos para mitigar los riesgos identificados.

Es por este motivo que se buscó determinar las características de los flujos de trabajo del Proceso Auditor. Como medio para determinar dichas características y para la comprensión de los procesos de la CGR, se utilizó el concepto de Arquitectura Empresarial, específicamente el dominio de Arquitectura de Negocio que como lo definen Arango y otros (2010) y Koznov y otros (2015), se encarga de la descripción de la estructura organizacional y de los procesos de negocio a través del modelamiento, que como manifiestan Bolaños y López (2012), es una técnica que permite captar las características principales del proceso, identificando su flujo y sus componentes para poder así comunicarlo con otros procesos, comprender las relaciones entre sus partes y conocer sus actores, responsables y demás artefactos que intervienen en su ejecución. Por otro lado 
y con el fin de analizar el modelo de procesos desarrollado, se utilizó la simulación como herramienta para representar el funcionamiento del mismo, que como afirman Dubey y Veeramani (2017) y Villanueva (2008), es un procedimiento que nos permite analizar el comportamiento del sistema bajo diferentes circunstancias, el análisis de todos los cambios posibles y sus consecuencias, es decir, reemplazando variables y analizando el desempeño de los procesos hasta obtener la mejor alternativa.

No obstante a lo anterior y teniendo en cuenta los planteamientos de Álvarez y Patiño (2015) en cuanto a la existencia de aspectos críticos y cambiantes que atañen a la Arquitectura Empresarial, se puede afirmar que esta debe evolucionar al mismo ritmo que las condiciones del negocio le impongan, partiendo de un modelo que de forma coordinada plasme la realidad presente de la organización y modele su visión futura. De la misma manera Bueno y Pineda (2015) indican que la Arquitectura Empresarial es cada vez más reconocida como una metodología para la adaptación de las estrategias de las organizaciones a través de la gestión del cambio y AL-Ghamdi (2017) y Henkel y Sneiders (2017) destacan la posición de la Arquitectura empresarial como clave para mejorar la calidad y la eficiencia de las organizaciones, proporcionando descripciones claras y concisas de lo que Boyd y otros (2011) han denominado como capacidades organizacionales. Es por este motivo que los cambios deben reflejarse en los modelos de arquitectura y si no sucede así, lo más seguro es que la misma fallará como modelo de alineación entre negocio y necesidades. Por lo anterior y para contrarrestar las mencionadas fallas, es necesario asegurar que se dimensionen de manera apropiada las necesidades de la organización y garantizar una adecuada evolución de su modelo de arquitectura.

\section{MARCO CONTEXTUAL}

En Colombia el ejercicio de Control Fiscal que realiza la Contraloría General de la República desde la Constitución Política de 1991, se divide en Control Fiscal Macro y Micro.

\section{Proceso de control Fiscal Macro}

Este consiste en el desarrollo por parte de la CGR de una evaluación global y de manera general del patrimonio público en cuanto a la auditoría que se le hace al balance general de la hacienda y el tesoro, la certificación de las finanzas públicas, el informe sobre la deuda pública y el informe sobre el estado de los recursos naturales y ambientales, cuyos resultados presenta el Contralor General de la República ante el Congreso en pleno.

\section{Proceso de Control Fiscal Micro}

En este proceso acorde con las normas de auditoría generalmente aceptadas vigentes, la política pública y la gestión y los resultados fiscales de los entes objeto de Control Fiscal y de los planes, programas, proyectos y/o asuntos a auditar, se busca determinar el cumplimiento de los principios de la gestión fiscal, en la prestación de servicios o provisión de bienes públicos, y en desarrollo de los fines constitucionales y legales del Estado, de manera que le permita a la CGR fundamentar sus hallazgos, conclusiones y recomendaciones como resultado del proceso de auditoría.

En el ejercicio de Control Fiscal Micro la CGR puede aplicar los sistemas de control establecidos, tales como control financiero, control de legalidad, control de gestión, control de resultados, la revisión de cuentas y la evaluación del sistema de control interno.

\section{Fase de planeación del proceso auditor}

Según los procedimientos establecidos en la Guía de Auditoría AUDITE de la CGR, el proceso auditor cuenta con tres fases, planeación, ejecución e informe; identificando a partir de un análisis de las entradas y salidas de cada una de las fases mencionadas, que la fase de planeación es clave para el cumplimiento de los objetivos de la auditoría, la cual comprende la elaboración del cronograma de actividades; el conocimiento en detalle del ente objeto de control fiscal o asunto a auditar; la evaluación de los controles que el ente objeto de control fiscal tiene diseñados para mitigar los riesgos identificados por la CGR en la matriz de riesgos, mediante la aplicación de pruebas de recorrido; la determinación de los criterios técnicos de evaluación, alcance y estrategia de auditoría; hasta la elaboración y aprobación del Plan de Trabajo y de los Programas de Auditoría.

\section{Arquitectura de Negocio}

Ordoñez y Otros (2014) mencionan que la Arquitectura de Negocio en las organizaciones es normalmente modelada o creada, utilizando herramientas para el diseño de procesos de negocio en donde plasman las operaciones o tareas que se necesita ejecutar en la organización. Con el propósito de determinar las 
características de los flujos de trabajo de la fase de planeación del proceso auditor, se utilizaron las bondades en cuanto a modelamiento que ofrece la Arquitectura de Negocio para comprender las relaciones entre sus partes y conocer sus actores, responsables y demás artefactos que intervienen en su ejecución.

A partir de los planteamientos anteriores y teniendo en cuenta lo argumentado por Arroyo (2015) en cuanto a que la Arquitectura de Negocio debe contar con herramientas para que la organización evolucione hacia un mejor estado futuro, se realizó el modelado y simulación utilizando una herramienta tecnológica BPSim (Business Process Simulación) bajo los estándares BPMN (notación gráfica creada para proveer un lenguaje unificado de acepción mundial) del OMG (Object Management Group 2011) y XPDL (formato estándar para el intercambio de definiciones de procesos de negocio entre diferentes productos) definido por la WfMC (Workflow Management Coalition 2005) que permiten visualizar el impacto de los cambios propuestos a los procesos empresariales antes de su implementación en el mundo real, como se afirma en Bizagi (2014) o en el Proceso Auditor, identificado como clave para el cumplimiento de la misión de la CGR.

\section{METODOLOGÍA}

El desarrollo metodológico se presenta en dos etapas: en una se describen las dimensiones para limitar el alcance de la arquitectura seleccionada para realizar la comprobación; y en otra etapa la aplicación del modelado y simulación a través de la herramienta de software.

\section{Etapa 1. Dimensiones para limitar el alcance de la arquitectura seleccionada}

Tomando como base el marco de referencia de arquitectura empresarial TOGAF Versión 9.1 (2011), según su metodología y lo descrito por Cerinza (2015), se identificaron las siguientes dimensiones para definir y limitar el alcance de la actividad de arquitectura, como se muestra en la tabla 1.

Tabla 1: Dimensiones para limitar el alcance de la arquitectura

\begin{tabular}{|l|l|}
\hline Dimensión & Consideraciones \\
\hline Amplitud & $\begin{array}{l}\text { ¿Cuál es la extensión total de la empresa, y con qué parte de } \\
\text { esa extensión debería tratar el esfuerzo de arquitectura? }\end{array}$ \\
\hline Profundidad & $\begin{array}{l}\text { ¿Qué nivel de detalle debería alcanzar el esfuerzo de } \\
\text { arquitectura? }\end{array}$ \\
\hline Dominios de arquitectura & ¿Cuál o cuáles dominios de arquitectura se van a especificar? \\
\hline
\end{tabular}

\section{Amplitud}

En cuanto a la extensión de la CGR para la actividad de arquitectura y debido a su tamaño, solo se tuvo en cuenta el macro proceso misional de control fiscal micro, tabla 2; en el cual según la guía de auditoria de la CGR, se realizan las tres fases del proceso de autoría; planeación, ejecución e informe, las cuales se describen con mayor detalle en la tabla 3.

Tabla 2: Descripción general del macro proceso de control fiscal micro

\begin{tabular}{|c|c|}
\hline \multicolumn{2}{|c|}{ Macro Proceso De Control Fiscal Micro } \\
\hline \multicolumn{2}{|c|}{ Objetivo } \\
\hline \multicolumn{2}{|c|}{$\begin{array}{l}\text { Vigilar la gestión fiscal y el control de resultados de las entidades y/o particulares que manejen fondos o bienes } \\
\text { públicos. }\end{array}$} \\
\hline Recursos & Normatividad \\
\hline $\begin{array}{l}\text { Talento humano, recursos financieros, infraestructura } \\
\text { de telecomunicaciones y sistemas de información. }\end{array}$ & $\begin{array}{l}\text { Entidades a vigilar (según resolución vigente), AUDITE, } \\
\text { Decreto } 267 \text { de 2000, Ley } 610 \text { de } 2001 \text { y Ley } 42 \text { de } \\
\text { 1993. }\end{array}$ \\
\hline \multicolumn{2}{|c|}{ Dependencias responsables } \\
\hline \multicolumn{2}{|c|}{$\begin{array}{l}\text { Contralorías Delegadas y Direcciones de Vigilancia Fiscal de los sectores: medio ambiente, agropecuario, } \\
\text { infraestructura, defensa justicia y seguridad, minas y energía, social, gestión pública y la Unidad de Investigaciones } \\
\text { Especiales para la Corrupción. }\end{array}$} \\
\hline \multicolumn{2}{|c|}{ Procesos relacionados con el macro proceso } \\
\hline
\end{tabular}


Tabla 3: Fases del proceso de auditoría

\begin{tabular}{|c|c|c|}
\hline Fase & Objetivo & Alcance \\
\hline $\begin{array}{l}\text { Fase de Planeación } \\
\text { de la Auditoría }\end{array}$ & $\begin{array}{l}\text { Definir el alcance y la estrategia } \\
\text { de auditoría a partir del } \\
\text { conocimiento del auditado y de } \\
\text { la evaluación de los controles } \\
\text { que el ente objeto de control } \\
\text { fiscal tiene establecidos para } \\
\text { mitigar los riesgos identificados } \\
\text { por la CGR en la matriz de } \\
\text { riesgos construidas por las } \\
\text { Unidades Ejecutoras. }\end{array}$ & $\begin{array}{l}\text { Esta fase se inicia con la asignación de actividades (asignación } \\
\text { de trabajo AT) y comprende: la elaboración del cronograma de } \\
\text { actividades para la fase de planeación; el conocimiento en } \\
\text { detalle del ente objeto de control fiscal o asunto a auditar; la } \\
\text { evaluación de los controles que el ente objeto de control fiscal } \\
\text { tiene diseñados para mitigar los riesgos identificados por la } \\
\text { CGR en la matriz de riesgos, mediante la aplicación de } \\
\text { pruebas de recorrido; la determinación de los criterios técnicos } \\
\text { de evaluación, alcance y estrategia de auditoría; hasta la } \\
\text { elaboración y aprobación del Plan de Trabajo y de los } \\
\text { Programas de Auditoría. }\end{array}$ \\
\hline $\begin{array}{l}\text { Fase de Ejecución } \\
\text { de la Auditoría }\end{array}$ & $\begin{array}{l}\text { La fase de ejecución es la parte } \\
\text { central de la auditoría, en ella se } \\
\text { practican todas las pruebas y se } \\
\text { utilizan todas las técnicas o } \\
\text { procedimientos para encontrar } \\
\text { las evidencias de auditoría que } \\
\text { sustentarán el informe. }\end{array}$ & $\begin{array}{l}\text { El análisis está orientado a examinar de acuerdo con el } \\
\text { alcance de la auditoría los siguientes aspectos, entre otros: } \\
\text { Obtención de los bienes y/o servicios que produce el auditado } \\
\text { en términos de calidad, cantidad, costo y oportunidad. } \\
\text { Evaluación de los riesgos de desvío de los recursos para la } \\
\text { obtención de los bienes y/o servicios. } \\
\text { Cumplimiento de la reglamentación que regula sus } \\
\text { operaciones. } \\
\text { Razonabilidad de la información financiera. } \\
\text { Calidad y eficiencia del control interno del auditado }\end{array}$ \\
\hline $\begin{array}{l}\text { Fase de Informe de } \\
\text { la Auditoría }\end{array}$ & $\begin{array}{l}\text { Determinar los parámetros y } \\
\text { directrices que se deben aplicar } \\
\text { en la fase de informe de la } \\
\text { auditoría, con el fin de } \\
\text { estructurar un documento con } \\
\text { los resultados del proceso } \\
\text { auditor que se comunica a los } \\
\text { auditados y destinatarios } \\
\text { correspondientes. }\end{array}$ & $\begin{array}{l}\text { Esta fase inicia con la revisión del Memorando de Preparación } \\
\text { de Resumen de Auditoría -MPRA (documento que en un } \\
\text { acápite incluye la totalidad de hallazgos u observaciones de } \\
\text { auditoría) y culmina con la firma y comunicación del informe de } \\
\text { auditoría. }\end{array}$ \\
\hline
\end{tabular}

\section{Profundidad}

Para tal fin se aplicaron los pasos del ciclo de vida bajo el enfoque BPM (Business Process Model) con el fin de entender los procesos de negocio de la CGR, dando como resultado que un punto neurálgico y del cual dependen el rumbo y los resultados del Proceso Auditor, es la fase de planeación. Dicha fase tiene como objetivo definir el alcance y la estrategia de auditoría a partir del conocimiento del auditado y de la evaluación de los controles para mitigar los riesgos, fig. 1.

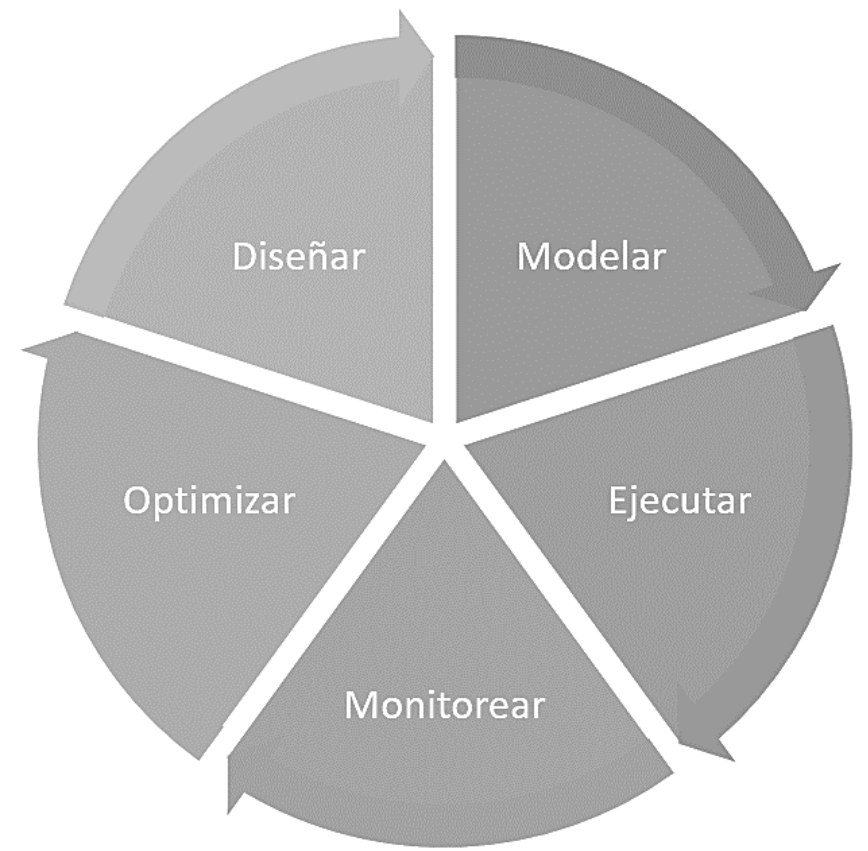

Fig. 1: Ciclo de vida bajo un enfoque BPM 


\section{Dominios de arquitectura}

Teniendo en cuenta que el marco de referencia TOGAF es modular, lo que admite particionar las arquitecturas dentro de una misma empresa o áreas de ésta por dominios, fue elegido el dominio de arquitectura de negocio, para lo cual se debe seleccionar una herramienta que permita definir un modelo e identificar las acciones que permitan mejorar su desempeño. De tal modo, la definición del proceso para el modelamiento y simulación se inició haciendo uso del dominio de arquitectura de negocio a nivel de diagnóstico, el cual implica realizar un análisis de la situación actual y un inventario de procesos de negocio, fig. 2.

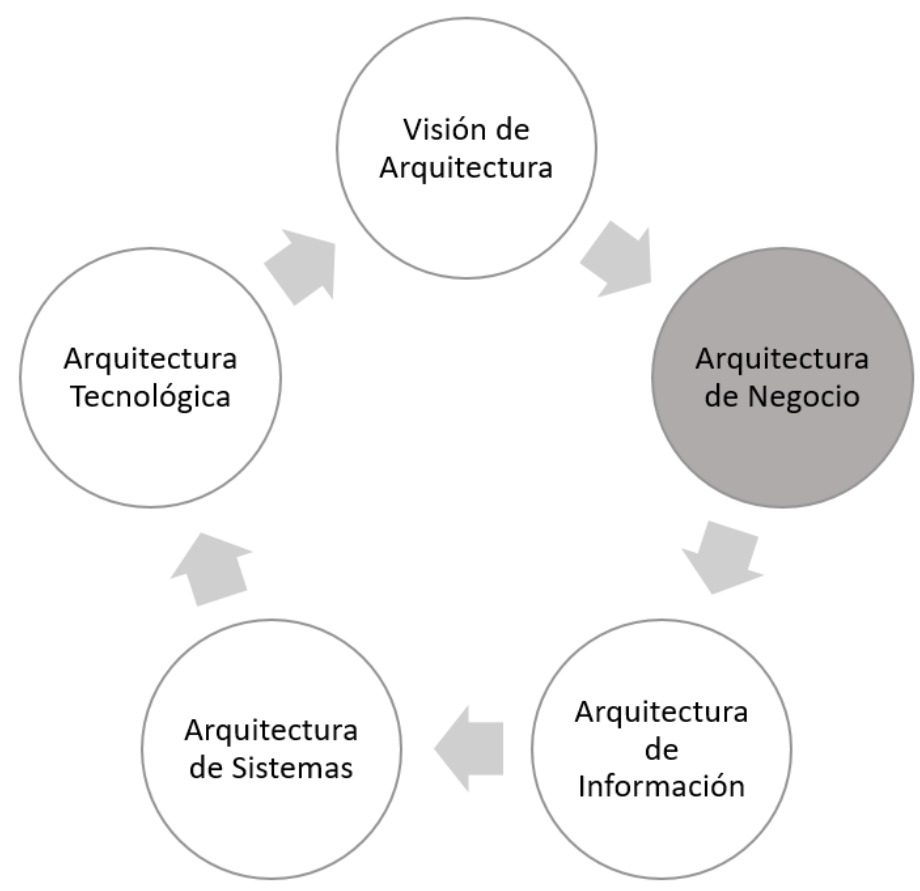

Fig. 2: Dominios de arquitectura según TOGAF

\section{Etapa 2. Aplicación del modelado y simulación a través de la herramienta}

Una vez detalladas las dimensiones que permitieron definir el alcance de la arquitectura para realizar la comprobación y con base en las actividades descritas en la guía de auditoría AUDITE de la CGR para la fase de planeación del Proceso Auditor, se realizó el modelo de procesos sobre el cual se ejecutó la simulación aplicando la metodología propuesta por Giraldo (2014), como se especifica a continuación.

\section{Formulación del problema}

Antes de la aplicación del modelado y simulación a través de la herramienta seleccionada, se realizó el análisis lógico del modelo de procesos con cada una de sus actividades y subprocesos, los diferentes roles y funciones de los involucrados dentro del mismo, la duración de las tareas y los recursos que se invierten para el desarrollo de éste. Por lo mismo se pudo evidenciar que el proceso inicia con la notificación del memorando de asignación y comprende la elaboración del cronograma de actividades para la misma; el análisis en detalle del ente o asunto a auditar; la evaluación del sistema de control interno o de los mecanismos de control; la determinación de los criterios técnicos de evaluación alcance y estrategia de auditoría, hasta la elaboración y aprobación del plan de trabajo y de los programas de auditoría. Del mismo modo se pudo comprobar que el comité técnico, el responsable de auditoría y el equipo auditor, son los roles del proceso y responsables de las tareas que se desarrollan al interior de éste. Como consecuencia de lo anterior y teniendo en cuenta que se evidencia interdependencia entre procesos y subprocesos, se exhibe variabilidad en su comportamiento y la relación costo, tiempo y beneficios, se puede justificar que una solución al problema planteado es experimentar con un modelo de procesos a través de la simulación y analizar sus resultados antes de tomar cualquier decisión con la arquitectura de la organización.

\section{Objetivos de la simulación}

Con base en lo anterior, se logra se identificar que los principales objetivos de la simulación son la determinación y eliminación de las tareas que generan cuellos de botella y reprocesos en la fase de planeación del proceso auditor, lo que puede implicar el rediseño estructural de éste, así como cambios en aspectos 
claves como la asignación de personal para las diferentes tareas de la auditoría y el número adecuado de personal a través de la configuración de variables que permitan mejorar el desempeño de toda la fase de planeación.

\section{Definición y conceptualización del modelo}

Previo a la ejecución de la simulación y después de haber realizado el análisis del proceso y subprocesos involucrados, se realizó la modelación haciendo uso del software Bizagi Process Modeler, el cual es una herramienta que permite modelar y documentar procesos de negocio bajo el estándar Business Process Model and Notation (BPMN), dando como resultado las actividades, tipo de elementos y notación que se describe en la tabla 4.

Tabla 4: Descripción de las actividades de la fase de Planeación del Proceso de Auditoría en notación BPMN

\begin{tabular}{|c|c|c|}
\hline Actividad y/o Subproceso & Tipo de elemento & Descripción y notación en BPMN \\
\hline Inicio & Evento de inicio & $\begin{array}{l}\text { Indica dónde se inicia un proceso al cumplir con una } \\
\text { condición específica. Para el modelo en estudio, éste } \\
\text { se activa al recibir la asignación de auditoría. }\end{array}$ \\
\hline $\begin{array}{l}\text { Definir cronograma de la fase de } \\
\text { planeación }\end{array}$ & Proceso & \multirow{4}{*}{$\begin{array}{l}\text { Son procesos o subprocesos cuyos detalles internos } \\
\text { han sido modelados utilizando actividades, } \\
\text { compuertas, eventos y flujos de secuencia. }\end{array}$} \\
\hline $\begin{array}{l}\text { Analizar en detalle el ente o asunto a } \\
\text { auditar }\end{array}$ & Proceso & \\
\hline Evaluar el sistema de control interno & Proceso & \\
\hline Determinar la estrategia de auditoría & Proceso & \\
\hline $\begin{array}{l}\text { Analizar el memorando de } \\
\text { asignación de auditoría }\end{array}$ & Tarea & \multirow{4}{*}{$\begin{array}{l}\text { Las tareas son actividades atómicas dentro de los } \\
\text { flujos de los procesos o subprocesos y se utilizaron } \\
\text { cuando las labores no se pudieron desglosar a un } \\
\text { nivel más bajo de detalle. }\end{array}$} \\
\hline Elaborar plan de trabajo & Tarea & \\
\hline $\begin{array}{l}\text { Determinar coherencia del plan de } \\
\text { trabajo }\end{array}$ & Tarea & \\
\hline Aprobación requerida & Tarea & \\
\hline ¿Se aprueba plan de trabajo? & Compuerta & \multirow[b]{2}{*}{$\begin{array}{l}\text { La compuerta se utilizó en el modelo para unir } \\
\text { caminos alternativos teniendo en cuenta una } \\
\text { condición para el flujo. }\end{array}$} \\
\hline $\begin{array}{l}\text { ¿Son coherentes los tiempos } \\
\text { establecidos? }\end{array}$ & Compuerta & \\
\hline Enfoque en controles & $\begin{array}{l}\text { Compuerta } \\
\text { exclusiva }\end{array}$ & \multirow[b]{2}{*}{$\begin{array}{l}\text { Para el modelo se utilizaron estas compuertas de } \\
\text { divergencia para crear caminos alternativos dentro } \\
\text { del flujo del proceso y seleccionando uno solo de ellos }\end{array}$} \\
\hline Enfoque sustantivo & $\begin{array}{l}\text { Compuerta } \\
\text { exclusiva }\end{array}$ & \\
\hline Fin & Evento de fin & $\begin{array}{l}\text { Con este evento se finaliza el proceso y todas sus } \\
\text { actividades. }\end{array}$ \\
\hline
\end{tabular}

\section{Modelo propuesto}

Haciendo uso del software Bizagi Process Modeler se construyó el modelo de procesos para la fase de planeación del proceso auditor, determinando las actividades y las decisiones que se toman dentro de los diferentes flujos de trabajo, lo que permitió definir los componentes que conforman el proceso, así como establecer la funcionalidad de sus relaciones y posibles mejoras al desempeño del proceso, Fig. 3. 


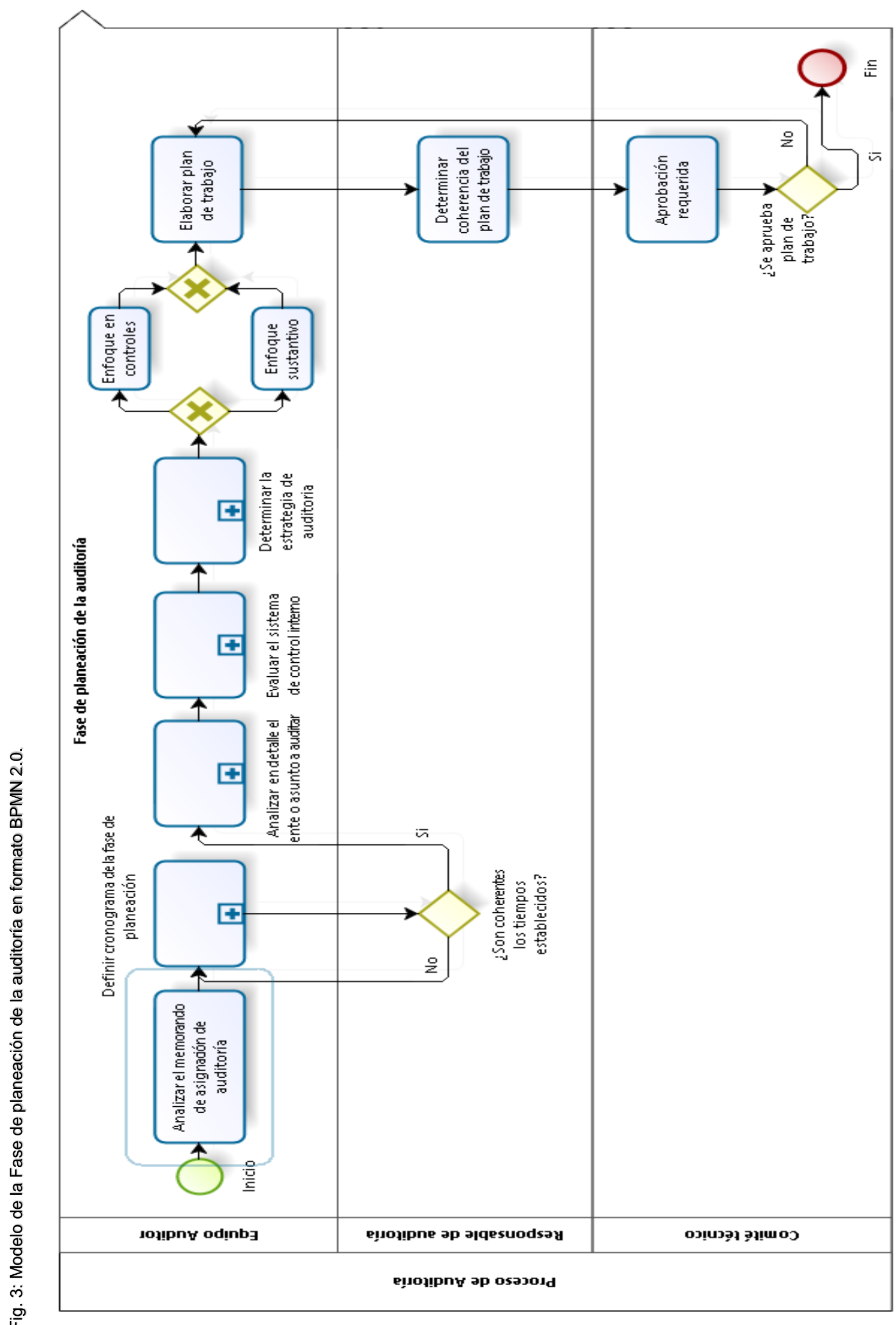




\section{Simulación del modelo de procesos}

A la hora de ejecutar la simulación de los flujos de trabajo corriendo los cuatro niveles, i) Validación; ii) Análisis de tiempo; iii) Análisis de recursos; iv) Análisis de calendarios, se nota que en cada nivel de la vista de simulación se añade mayor grado de detalle al modelo, la posibilidad de crear escenarios para distintas combinaciones de datos y analizar sus resultados en busca de posibles mejoras al desempeño del proceso, para lo cual se tienen que aplicar los pasos sugeridos por Bizagi (2014), fig. 4.

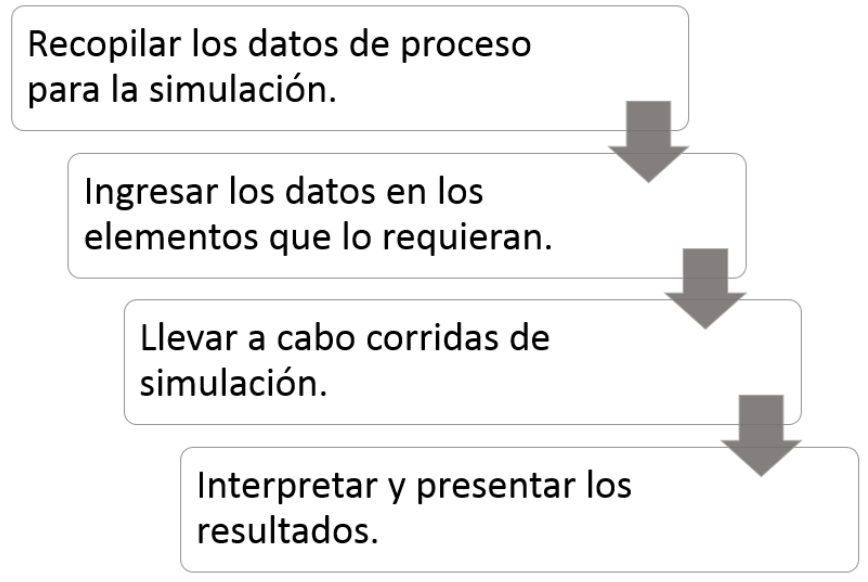

Fig. 4: Pasos para cada nivel de simulación

\section{Validación del proceso}

Después de diseñar el modelo se procedió a realizar su validación, que como lo menciona Stary (2014) es crucial para verificar la coherencia del mismo. En la herramienta este nivel se ejecuta con el fin de evaluar la estructura y la lógica del diagrama desarrollado, tabla 5; es por esto que se programaron las compuertas de decisión con las probabilidades correspondientes a cada uno de los flujos de proceso.

Tabla 5: Nivel de validación basado en Bizagi (2014)

\begin{tabular}{|l|l|l|}
\hline Definición & Datos & Resultados \\
\hline La simulación básica & Se requiere la información de & Los resultados muestran si se activan \\
para evaluar la & $\begin{array}{l}\text { porcentaje estimado para los flujos } \\
\text { todos los caminos del proceso y se } \\
\text { estructura del diagrama } \\
\text { de secuencia de compuertas, para } \\
\text { de proceso. }\end{array}$ & $\begin{array}{l}\text { asegura que todas las instancias creadas } \\
\text { en realidad sean finalizadas. Además }\end{array}$ \\
& $\begin{array}{l}\text { así como la información de la } \\
\text { activación de instancias del } \\
\text { proceso, en el Evento de Inicio. }\end{array}$ & $\begin{array}{l}\text { pasan el número de instancias que } \\
\text { secuencia, actividad y evento final. }\end{array}$ \\
\hline
\end{tabular}

Al iniciar la validación se configuran los datos de entrada en el evento de inicio, en este caso con 25 instancias de tokens, fig 5; lo que equivale a la planeación de 25 auditorías. En tanto al enrutamiento de las compuertas, para la compuerta "Son coherentes los tiempos establecidos" y "Se aprueba plan de trabajo" se tiene un 50\% de posibilidades que la respuesta sea "No" y un $50 \%$ de posibilidades que sea "Si". Por otra parte para la compuerta "Determinar la estrategia de auditoría" se tiene un 95\% de posibilidades que el flujo de trabajo vaya por el "Enfoque en controles" y el $5 \%$ que sea "Enfoque sustantivo" fig, 6 . Estos porcentajes se obtuvieron de distribuciones históricas de auditorías realizadas en anteriores PGA (Plan General de Auditoría de la CGR) bajo variadas condiciones.

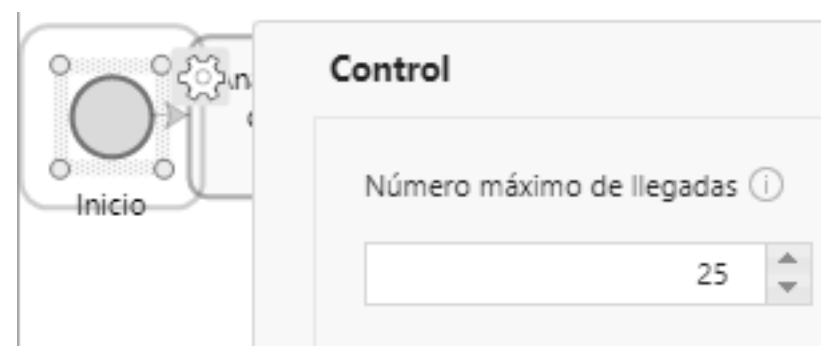

Fig. 5: Configuración de tokens 


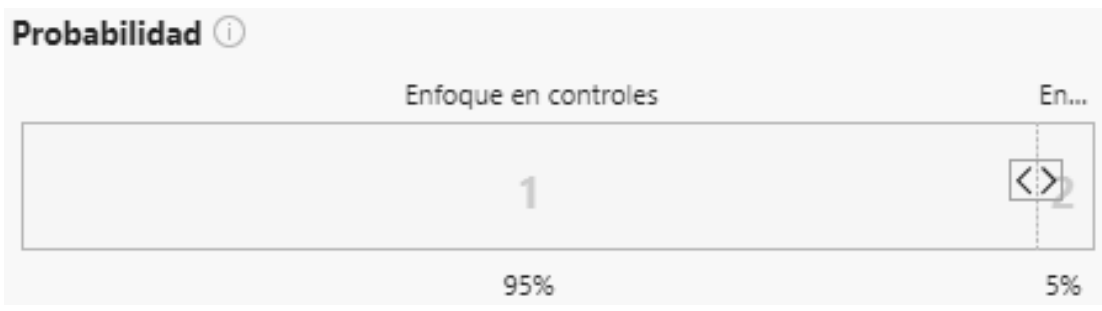

Fig. 6: Validación del modelo a través de compuertas

En el análisis de los resultados se puede concluir que el modelo es correcto, ya que se activan todos los caminos del proceso y se asegura que todas las instancias creadas (25) en realidad sean finalizadas, tabla 6. Así mismo se puede observar que las actividades que generan un mayor número de re procesos son las de tipo tarea con 64 instancias de tokens.

Tabla 6: Resultados nivel de validación

\begin{tabular}{|l|l|c|}
\hline Nombre & Tipo & Instancias completadas \\
\hline Analizar el memorando de asignación de auditoría & Tarea & 25 \\
\hline Elaborar plan de trabajo & Tarea & 64 \\
\hline Determinar coherencia del plan de trabajo & Tarea & 64 \\
\hline ¿Se aprueba plan de trabajo? & Compuerta & 64 \\
\hline Aprobación requerida & Tarea & 36 \\
\hline ¿Son coherentes los tiempos establecidos? & Compuerta & 24 \\
\hline Enfoque en controles & Compuerta & 25 \\
\hline Enfoque sustantivo & Compuerta & 25 \\
\hline Fin & Evento de Fin & 36 \\
\hline Inicio & Evento de inicio & 25 \\
\hline Definir cronograma de la fase de planeación & Proceso & 25 \\
\hline Analizar en detalle el ente o asunto a auditar & Proceso & \\
\hline Evaluar el sistema de control interno & Proceso & \\
\hline
\end{tabular}

\section{Análisis de tiempo}

Este nivel se simula para comprender el tiempo de procesamiento de cada actividad y el intervalo de tiempo entre la generación de las instancias del proceso. Los resultados deben mostrar el desempeño del proceso de planeación de auditoría, consolidando los tiempos mínimos, máximos, medios y totales de procesamiento, para lo cual se establece la duración de cada tarea y subproceso asignándole valores determinísticos o probabilísticos a los tiempos, según ocurre en la realidad. Para el modelo el tiempo del subproceso "Elaborar plan de trabajo" se distribuye probabilísticamente mediante una distribución normal, fig. 7.

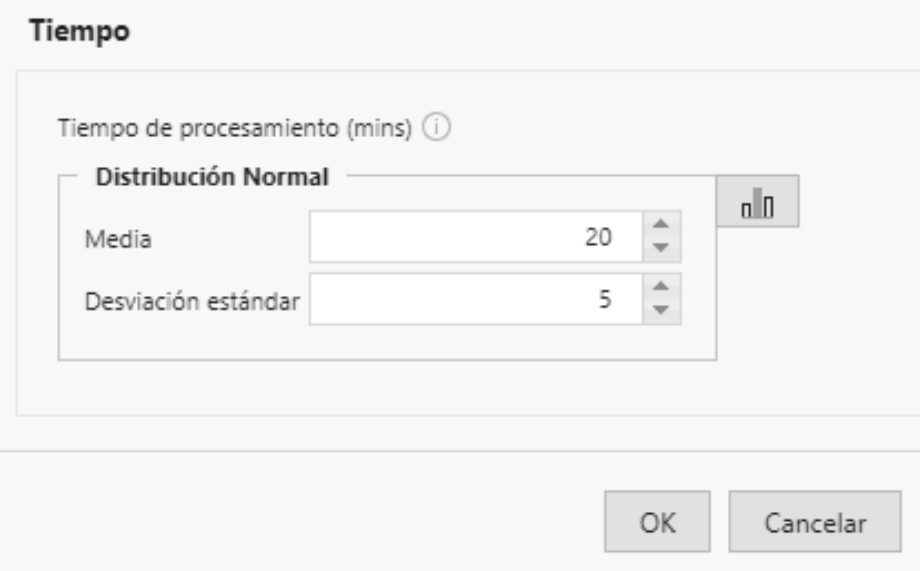

Fig.7: Nivel de análisis de tiempo 
Una vez todos los tiempos de procesamiento se han definido y después de ejecutar la simulación, a través de las herramientas de análisis se pueden identificar el número de instancias completadas, el tiempo promedio por actividad y el tiempo total de procesamiento, resultados que se discutirán en el siguiente aparte.

\section{Análisis de recursos}

Adicional a los datos introducidos en los niveles anteriores, en este nivel se incluyen los recursos disponibles, tales como costo y personal. Como objetivo este nivel pretende identificar y minimizar el impacto de estas limitaciones de tiempo de ciclo y costos. Teniendo en cuenta que el recurso más valioso para el modelo, es el personal conformado por los equipos de auditores, se realiza la asignación correspondiente, fig. 8 y fig. 9.

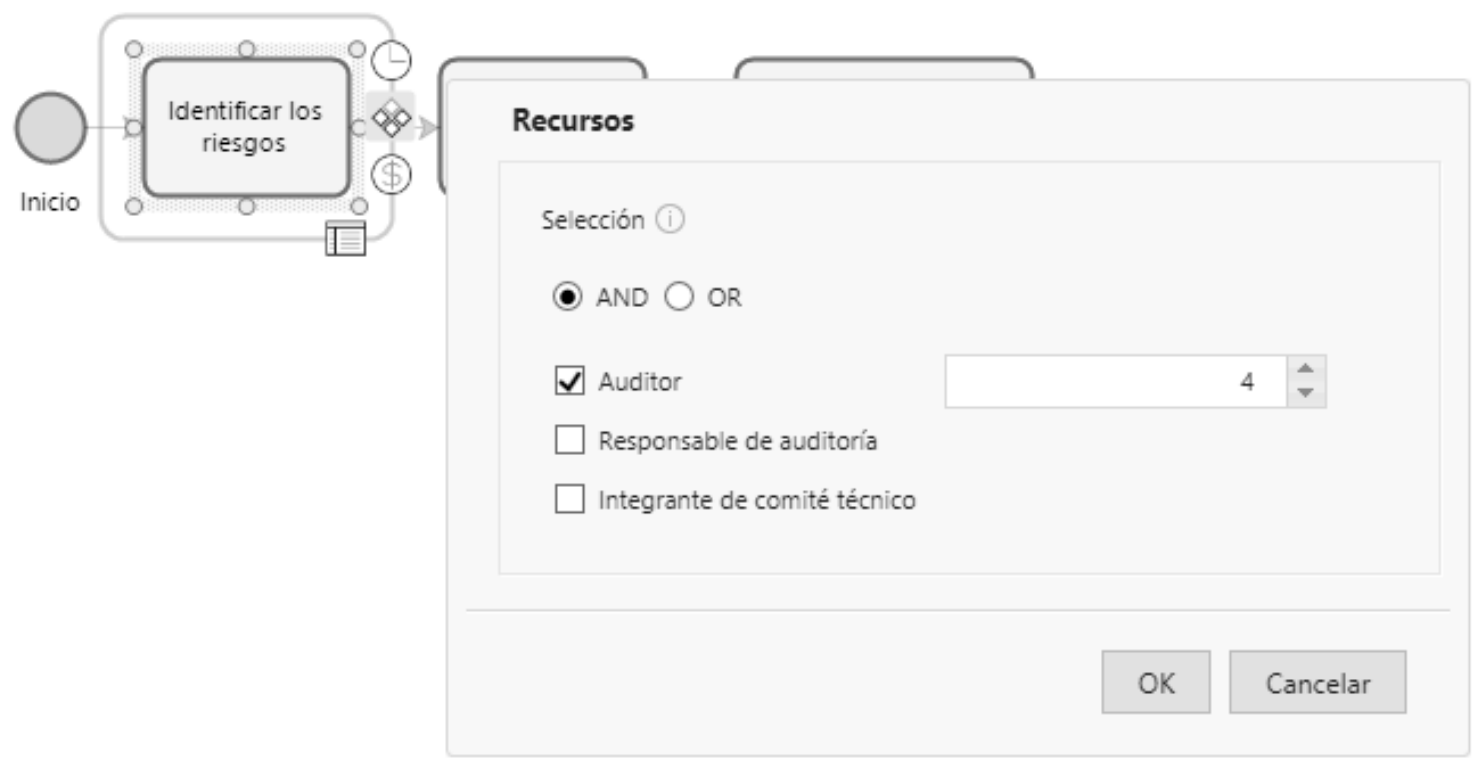

Fig. 8: Nivel de análisis de recursos

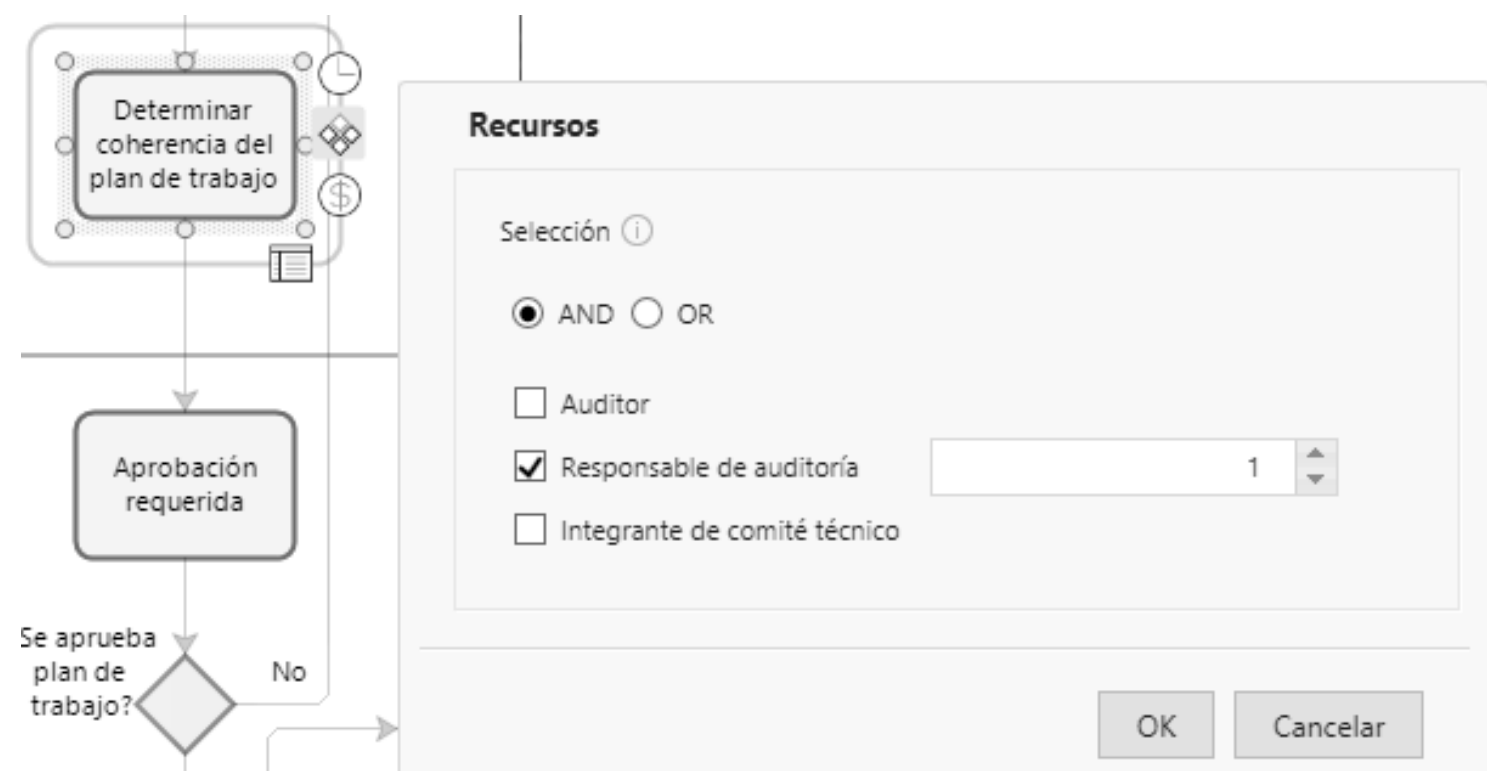

Fig. 9: Nivel de análisis de recursos

\section{Análisis de Calendarios}

En cuanto a este nivel, se obtiene como resultado que la disponibilidad de tiempo por parte de los auditores, en diferentes etapas del proceso es exagerada, lo que se debe a la espera de aprobaciones por parte de instancias superiores, impidiendo que el flujo continúe hasta que se obtenga una respuesta positiva. En este nivel se definen los calendarios de los procesos en periodos de tiempo, que para este caso corresponde a la duración de la etapa de planeación del proceso auditor con su patrón y rango de recurrencia, fig. 10. 


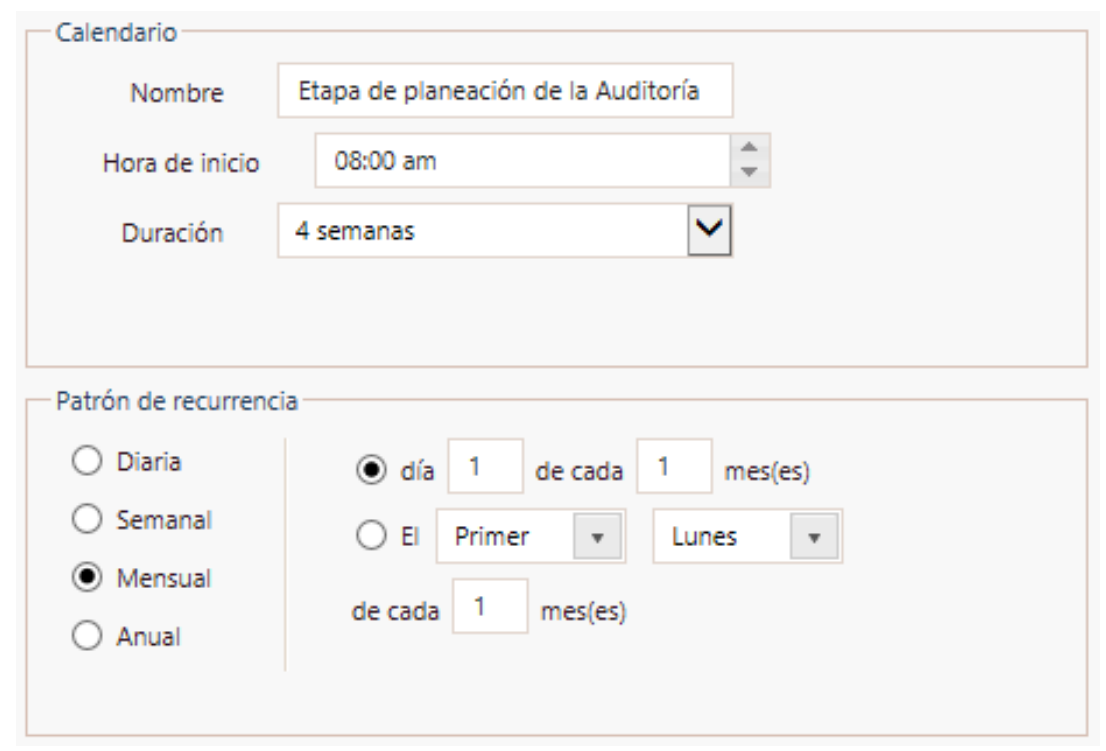

Fig. 10: Nivel de análisis de calendarios

\section{RESULTADOS Y DISCUSIÓN}

Al ejecutar el nivel de análisis de tiempo, simulando 25 instancias correspondientes al mismo número de auditorías, se obtiene como resultado la duración de cada una de las tareas, así como el tiempo total en que estas estuvieron en funcionamiento. Como se puede evidenciar en estos resultados, los tiempos no son coherentes con los trabajos desarrollados, lo que se puede justificar por la rigidez del proceso al depender de la aprobación del responsable de auditoría y del comité técnico, generando un cuello de botella en los flujos de trabajo, lo que puede en un momento determinado entorpecer el inicio de las otras fases del proceso auditor, como la de ejecución y hasta el mismo informe de auditoría.

Como consecuencia de lo anterior y teniendo en cuenta la evolución del modelo de Arquitectura Empresarial, se crearon tres escenarios para analizar el comportamiento al realizar cambios en el proceso, el primer escenario es configurado con datos que se obtuvieron de distribuciones históricas de auditorías anteriores (escenario actual), un segundo escenario (escenario transitorio), modificando el reproceso "Aprobación requerida" y un tercer escenario (escenario objetivo), en el que se eliminó el reproceso que generaba un cuello de botella en los flujos de trabajo al depender de dos instancias, asignándole la aprobación al responsable de auditoría cuando estas se presentan de manera repetitiva por falta de coherencia en su planteamiento, observándose una reducción del $29.8 \%$, en el tiempo total del proceso, tabla 7.

Tabla 7: Resultados de simulación - Validación de proceso y análisis de tiempo

\begin{tabular}{|c|c|c|c|c|c|c|}
\hline \multirow[t]{2}{*}{ Nombre proceso } & \multicolumn{2}{|c|}{ Escenario actual } & \multicolumn{2}{|c|}{ Escenario transitorio } & \multicolumn{2}{|c|}{ Escenario objetivo } \\
\hline & Instancias & Tiempo $(d)$ & Instancias & Tiempo $(d)$ & Instancias & Tiempo (d) \\
\hline Proceso de Auditoría & 25 & 33.5 & 25 & 28.5 & 25 & 23.5 \\
\hline $\begin{array}{l}\text { Analizar el memorando de } \\
\text { asignación de auditoría }\end{array}$ & 25 & 1 & 25 & 1 & 25 & 1 \\
\hline Elaborar plan de trabajo & 59 & 5 & 59 & 5 & 59 & 5 \\
\hline $\begin{array}{l}\text { Determinar coherencia del plan } \\
\text { de trabajo }\end{array}$ & 59 & 5 & 59 & 5 & 59 & 5 \\
\hline ¿Se aprueba plan de trabajo? & 59 & & 59 & & 59 & \\
\hline Aprobación requerida & 59 & 10 & 59 & 5 & 59 & 0 \\
\hline $\begin{array}{l}\text { ¿Son coherentes los tiempos } \\
\text { establecidos? }\end{array}$ & 40 & & 40 & & 40 & \\
\hline Compuerta exclusiva & 25 & & 25 & & 25 & \\
\hline Enfoque en controles & 15 & 1 & 15 & 1 & 15 & 1 \\
\hline Enfoque sustantivo & 10 & 0 & 10 & 0 & 10 & 0 \\
\hline Compuerta exclusiva & 25 & & 25 & & 25 & \\
\hline Fin & 25 & & 25 & & 25 & \\
\hline
\end{tabular}


Tabla 7 (continuación)

\begin{tabular}{|l|c|c|c|c|c|c|}
\hline Inicio & 25 & & 25 & 25 & 40 \\
\hline $\begin{array}{l}\text { Definir cronograma de la fase de } \\
\text { planeación }\end{array}$ & 40 & 0.5 & 40 & 0.5 & 25 & 5 \\
\hline $\begin{array}{l}\text { Analizar en detalle el ente o } \\
\text { asunto a auditar }\end{array}$ & 25 & 5 & 25 & 5 & 25 & 3 \\
\hline $\begin{array}{l}\text { Evaluar el sistema de control } \\
\text { interno }\end{array}$ & 25 & 3 & 25 & 3 & 25 & 3 \\
\hline $\begin{array}{l}\text { Determinar la estrategia de } \\
\text { auditoria }\end{array}$ & 25 & 3 & 25 & 3 & 3 \\
\hline
\end{tabular}

Otro aspecto importante que se logro identificar en los resultados de la simulación es la relación entre los flujos de trabajo y los recursos utilizados, fig. 11; donde se observa que el aprovechamiento del recurso auditor teniendo en cuenta los escenarios ya planteados, paso de 9,4\% a $13,4 \%$, por el contrario para los integrantes del comité técnico, disminuyo su ustilización de 3,3\% a 0,2\%. Con lo que se evidencia que al realizar los cambios planteados con miras a construir un escenario objetivo que mejore el desempeño del proceso, se logra también un aprovechamiento de los auditores como los principales protagonistas del desarrollo del mismo.

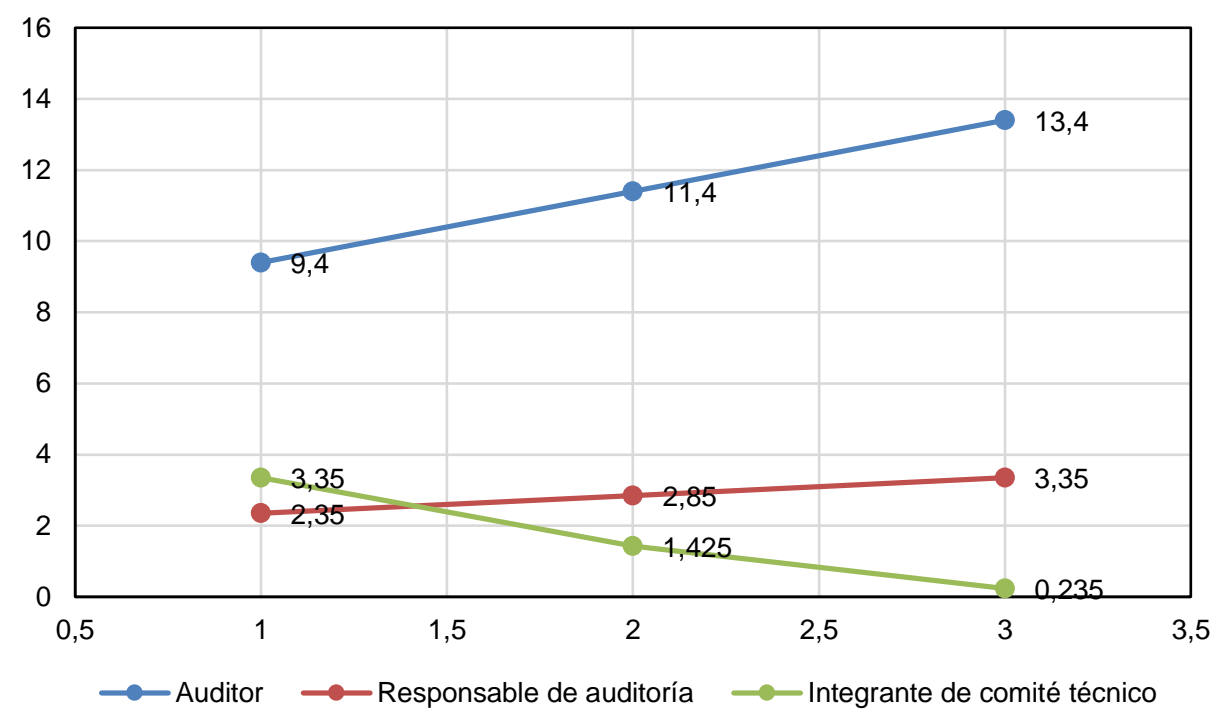

Fig. 11: Uso de recursos

Finalmente y partiendo de la idea que la construcción de una Arquitectura Empresarial se fundamenta en la planeación estratégica, las unidades organizativas, los roles y los procesos de negocio, Llenera (2015) y que para ello se debe partir de un modelo que de forma coordinada plasme la realidad presente de la organización y modele su visión futura, se evidencia la importancia de contar con herramientas que permitan probar los diferentes escenarios, para con ello controlar la evolución del esfuerzo de Arquitectura que realice la organización, fig. 12.

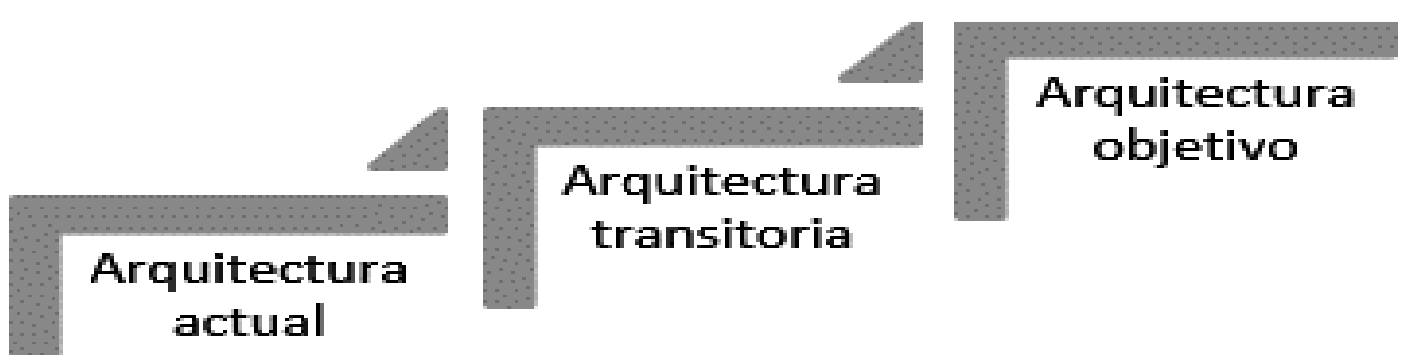

Fig. 12: Evolución de un modelo de arquitectura empresarial 


\section{CONCLUSIONES}

De los resultados expuestos en el punto anterior se pueden exponer las siguientes conclusiones:

(i) la construcción de una Arquitectura Empresarial es relevante, cuando ésta permite entender todos los elementos que intervienen en el modelo de la organización y en éste se pueden identificar varias alternativas o escenarios para elegir el mejor de ellos;

(ii) el estudio de caso aplicado a la CGR revela el valor de la fusión entre Arquitectura Empresarial y Simulación de Procesos, ya que los tiempos de procesamiento se pueden reducir debido a la ejecución de escenarios específicos y ajustados a las necesidades de desempeño;

(iii) la fusión con la Simulación de Procesos, genera la posibilidad de crear escenarios para distintas combinaciones de datos, garantizando una adecuada evolución desde un modelo actual a un modelo objetivo y permitiendo analizar sus resultados antes de tomar cualquier decisión con la arquitectura sin afectar a toda la organización en caso de realizar modificaciones;

(iv) el aporte más importante del modelo desarrollado fue la posibilidad de evidenciar la variabilidad e interdependencia entre procesos y subprocesos, así como la variabilidad en su comportamiento y la relación costo, tiempo y beneficios que justifica experimentar a través de la simulación y analizar sus resultados antes de tomar cualquier decisión con la arquitectura de la organización; y

(v) finalmente, se considera pertinente iniciar nuevas investigaciones donde no solamente se modele y simule el Proceso Auditor, sino otros como complemento a la construcción de un prototipo de arquitectura empresarial de los procesos misionales de la CGR.

\section{AGRADECIMIENTOS}

Los autores agradecen a la Universidad Nacional de Colombia sede Manizales, por el apoyo institucional y financiero al desarrollo de esta investigación, la cual hace parte del proyecto "La Arquitectura Empresarial como Instrumento para Analizar la Incidencia de la Administración con Enfoque Burocrático en los Resultados del Control Fiscal Micro. Caso aplicado", código HERMES 35472.

\section{REFERENCIAS}

Acosta, A. P. y M. M. Espino, Modelos de requisitos basados en i $^{\star}$ para detectar proactividad en dashboards, ISSN: 21454086, Rev. Lámpsakos, (12), 101-109 (2014)

AL-Ghamdi, A.S.A.M., A proposed model to measure the impact of business architecture, Cogent Business \& Management, 4(1), 1405493 (2017)

Álvarez, A. y A. Patiño, Enterprise architecture and agile methodologies-An effective combination to tackle the frequent business changes, ISSN: 2463-0128, Rev. Actas de Ingeniería, 1, 145-152 (2015)

Arango, M.D., J.E. Londoño y J.A. Zapata, Arquitectura empresarial - una visión general, ISSN: 1692-3324, Rev. Ingenierías, 9(16), 101-111 (2010)

Arroyo, E., Definición de principios de arquitectura para arquitectura empresarial de la organización, Handbook TI, 35 (2015)

Barros, O., Ingeniería de negocios. Diseño integrado de negocios, procesos y aplicaciones TI. Primera parte. Versión, 4. CEGES, Departamento de Ingeniería Industrial (2011)

Bizagi, Bizagi BPM Suite Functional Description, (en línea), (2015)

Bolaños, S.J. y S.A. López, LMPS como propuesta alterna a BPMN para el modelado de procesos de software, ISSN: 0123-921X, Rev. Tecnura, 16(34), 157-170 (2012)

Bork, D. y H.G. Fill, Formal aspects of enterprise modeling methods: a comparison framework. In System Sciences (HICSS), 47th Hawaii International Conference on (pp. 3400-3409), IEEE, Hawaii, USA, 6-9 de Enero (2014)

Boyd, L.B., S.P. Hunicke y otros cinco autores, The caBIG® life science business architecture model, Bioinformatics, 27(10), 1429-1435 (2011)

Bueno, H. C. y W. B. Pineda, Identificación de principios de arquitectura empresarial para la gestión de factores de impacto en entidades públicas colombianas utilizando TOGAF, Rev. INVENTUM, 10(18), $22-28$ (2015)

Cáceres, C.E. y O.M. Zea, Propuesta de un marco de referencia de gestión de organizaciones usando Arquitectura Empresarial, Enfoque UTE, 5(4), 70 (2014)

Cerinza, M.D.R., Modelo para medir los beneficios de una arquitectura empresarial, Tesis de Maestría, Escuela Colombiana de Ingeniería Julio Garavito, Bogotá, Colombia (2015) 
CGR - Contraloría General de la República, Contextualización de la Contraloría General de la República, Plan Anticorrupción y de Atención al Ciudadano - PAAC (2017)

CGR - Contraloría General de la República, Control Fiscal Participativo, Economía Colombiana, Ed. 315, enero-febrero (2016)

Crespo, P. y V. Santos, Construction of integrated business management systems for micro and small enterprises/Construcao de sistemas integrados de gestao para micro e pequenas empresas, RISTI (Revista Iberica de Sistemas e Tecnologias de Informacao), (15), 35-50 (2015)

Cuenca, L., A. Ortiz y A. Boza, Desarrollo de una Herramienta Software para la Vista de Información de la Arquitectura CIMOSA, Información Tecnológica, 19(3), 97-106 (2008)

Dubey, V.K. y D. Veeramani, A framework for sizing an automated distribution center in a retail supply chain, Simulation Modelling Practice and Theory, 75, 113-126 (2017)

Giraldo, J. A., Simulación de sistemas de producción y de servicios, $1^{\text {a }}$ Ed., Editorial Blanecolor, Manizales (2014)

Geyer, R.W. y C.J. Fourie, Determining the suitability of a business process modelling technique for a particular application, ISSN: 1012-277X, Rev. South African Journal of Industrial Engineering, 26(1), 252-266 (2015)

Grant, D., Business analysis techniques in business reengineering, Business Process Management Journal, 22(1), 7588 (2016)

Henkel, M., E. Perjons y E. Sneiders, Examining the potential of language technologies in public organizations by means of a business and IT architecture model, International Journal of Information Management, 37(1), 1507-1516 (2017)

Kotusev, S., Enterprise Architecture: What Did We Study? International Journal of Cooperative Information Systems, 26(04), 1730002 (2017)

Koznov, D.V., M.Y. Arzumanyan y otros tres autores, Specifics of projects in the area of Enterprise Architecture development, Business Informatics, 4 (34), 15-23 (2015)

Lange, M., J. Mendling y J. Recker, An empirical analysis of the factors and measures of Enterprise Architecture Management success, European Journal of Information Systems, 25(5), 411-431 (2016)

Llerena F.R., Definición de una estrategia de transición de la arquitectura empresarial en un entorno industrialbiotecnológico, ISSN: 2227-1899, Revista Cubana de Ciencias Informáticas, 9(3), 1-17 (2015)

OMG - Object Management Group, Business Process Model and Notation (BPMN), (en línea), (2015)

Ordoñez, H., J. C. Corrales y C. Cobos, MultiSearchBP: Entorno para búsqueda y agrupación de modelos de procesos de negocio, Polibits, (49), 29-38 (2014)

Peterson, H., Merging management ideals in Swedish IT offshoring, Scandinavian J. of Management, 32(2), 97-105 (2016)

Petro, Y. y P. Gardiner, An investigation of the influence of organizational design on project portfolio success, effectiveness and business efficiency for project-based organizations, International J. of Project Management, 33(8), 1717-1729 (2015)

Plattner, H., The impact of columnar in-memory databases on enterprise systems: implications of eliminating transactionmaintained aggregates, Proceedings of the VLDB Endowment, 7(13), 1722-1729 (2014)

Pylypenko, A. y A. Lytvynenko, Institutional and architectural design of organizational development of large-scale economic and industrial systems, Economic Annals-XXI, 165 (2017)

Restrepo, J.C.R., Modelos de gestión pública y organizaciones públicas un análisis de dependencia, ISSN: 2314-1662, Rev. Difusiones, 2(2) (2017)

Stary, C., Non-disruptive knowledge and business processing in knowledge life cycles-aligning value network analysis to process management, Journal of Knowledge Management, 18(4), 651-686 (2014)

The Open Group, Togaf 9.1. - Gruía (Latin American Spanish), (en línea), (2015)

Villanueva, J., La simulación de procesos, clave en la toma de decisiones, Rev. Dyna Ingeniería e Industria, 83(4), 221$227(2008)$

WfMC - Workflow Management Coalition, Process Definition Interface, (en línea), (2015) 
